\title{
Influências climáticas na produção de serapilheira em um cerradão em Prata - MG
}

\author{
Thiago Mendes Siqueira \\ Marcelo Henrique Ongaro Pinheiro* \\ Diego Gonçalves da Silva \\ Talita Moura Franco
}

Universidade Federal de Uberlândia, campus Pontal

Rua 20, 1600, Bairro Tupã, CEP 38304-402, Ituiutaba - MG, Brasil

* Autor para correspondência

mhop@pontal.ufu.br

Submetido em 24/09/2015

Aceito para publicação em 14/01/2016

\section{Resumo}

O estudo teve como objetivo principal analisar a influência climática na produção de serapilheira, em um pequeno fragmento de cerradão em Minas Gerais, Brasil, durante um ano. Foram utilizados 20 coletores $(0,25$ $\mathrm{m}^{2}$ ), dispostos em linha. A produção de serapilheira total foi de 7,5 tha ${ }^{-1} \cdot$ ano $^{-1}$, composta pelas frações folhas, ramos e estruturas férteis. A fração foliar contribuiu com $67 \%$ do total acumulado em um ano. A correlação de Pearson $(r)$ indicou a influência das variáveis climáticas precipitação e temperaturas médias e mínimas na produção da fração foliar. A análise de $r$ resultou também em valor significativo entre ramos e velocidade média do vento, enquanto que estruturas férteis sofreram influência da precipitação, temperaturas médias máximas e mínimas. Características vegetacionais podem ter interferido nos resultados encontrados. Todavia, estudos complementares envolvendo análises fitossociológica e de ciclagem de nutrientes deverão ser desenvolvidos para a comprovação de hipóteses propostas.

Palavras-chave: Aporte de folhas; Domínio cerrado; Formação savânica; Microclima

\section{Abstract}

Climatic influences on litterfall production in a forested savannah in Prata - MG. The objective of this study was to analyze the influence of climate on litterfall production, for a year, in a small fragment of forested savannah, in Minas Gerais, Brazil. Twenty collectors $\left(0.25 \mathrm{~m}^{2}\right)$ arranged in a row were used. The total litter production was $7.5 \mathrm{t}^{\mathrm{h} h \mathrm{~h}^{-1}}$.year ${ }^{-1}$, comprising leaves, branches and fertile structures as the fractions. The leaf fraction contributed $67 \%$ of the annual litter input. Pearson's correlation coefficient $(r)$ indicated the influence of the climatic variables precipitation and mean and minimum temperature in the accumulation of this fraction. The analysis also resulted in a significant $r$ value between branches and average wind velocity, while the fertile structure fraction was influenced by rainfall and average maximum and minimum temperature. Vegetation characteristics may have interfered with the results. Phytosociological and nutrient cycling studies should be conducted to test the proposed hypothesis.

Key words: Cerrado domain; Leaf input; Microclimate; Savanna formation 


\section{Introdução}

O aporte e decomposição de serapilheira são importantes processos relacionados à dinâmica de nutrientes nos ecossistemas, mantendo relação direta com a disponibilidade de nutrientes edáficos para os vegetais (COLEMAN et al., 2004; BERG; MCCLAUGHERTY, 2008). Apesar disso, esses processos ainda são pouco compreendidos no cerrado (CIANCIARUSO et al., 2006). Sobre essa afirmação, salienta-se que o número de trabalhos envolvendo estudos sobre ciclagem de nutrientes no cerrado, considerando a proporção das demais pesquisas ecológicas desenvolvidas nesse bioma, ainda é pequeno (OLIVEIRA; MARQUIS, 2002). Entretanto, sabe-se que esses processos, o acúmulo e degradação de serapilheira, sofrem notável influência da sazonalidade climática (CHAPIN et al., 2002), assim como de variações microambientais, as quais interferem na atividade de organismos decompositores, a partir de variações de umidade e temperatura da serapilheira (ADL, 2003; COLEMAN et al., 2004; RIUTTA et al., 2012).

O chamado efeito de borda, por sua vez, é um fenômeno capaz de interferir no aporte de serapilheira, ao definir diferenças na produção de frações da serapilheira total, e.g. folhas, gravetos, flores e frutos, entre as regiões de borda e central, em fragmentos vegetais (DIDHAM; LAWTON, 1999; SCHESSL et al., 2008). Deve ser mencionado ainda que a influência de agentes bióticos e abióticos, e.g., herbivoria e variação de nutrientes edáficos, são fatores capazes de alterar a produção de serapilheira em formações vegetais (LATHWELL; GROVE, 1986; CHOUDHURY, 1988). Em escala local, gradientes edáficos, de componentes físicos e químicos do solo, podem originar diferenças no volume e na qualidade química da serapilheira acumulada em comunidades vegetais (DENT et al., 2006).

A produção de serapilheira também sofre influência de animais de grande porte, como ungulados, através do pisoteio e pelo deslocamento no interior de formações vegetais, podendo alterar características edáficas, e.g. compactação do solo, estruturais e florísticas de comunidades vegetais (DAUBENMIRE, 1974; BARNES et al., 1998; DANELL; BERGSTRÖM, 2002). Esse fenômeno pode ser compreendido pelo seu potencial em originar distúrbios em comunidades vegetais, produzindo diferenças microambientais por meio de modificações localizadas na estrutura vegetacional(DANELL; BERGSTRÖM, 2002; BEGON et al., 2006). O impacto do pisoteio de gado bovino contribui para a permanência de vegetais de menor porte, como plântulas e vegetais herbáceo-arbustivos, assim como pela perda de parte de vegetais de maior porte, por razão do deslocamento desses animais em vegetação mais densa (DAUBENMIRE, 1974). Por conseguinte, ao definir diferenças na estrutura vegetacional, o distúrbio ambiental ocasionado por animais de maior porte, como gado bovino, interfere com o acúmulo da serapilheira em escala local (BARNES et al., 1998).

Salienta-se ainda que a produção de serapilheira sofre grande influência da fenologia de populações e comunidades vegetais, e ambos os processos devem ser compreendidos como mecanismos diretamente relacionados. Não obstante, os fatores bióticos e abióticos que interferem na manifestação de fenofases, como a sazonalidade climática, grau de perturbação e características estruturais de comunidades vegetais (VAN SCHAIK et al., 1993; WERNECK et al., 2001), acabam por determinar intensidades de produção da serapilheira total e de suas frações, i.e., folhas, flores, frutos, sementes. Características fenológicas distintas, entre as espécies vegetais (GUREVITCH et al., 2009), influenciadas por respostas diferenciadas a fatores acima descritos, podem aumentar, sobremaneira, a complexidade da dinâmica envolvendo produção e decomposição de serapilheira em diferentes formações vegetacionais.

Este estudo objetivou, principalmente, responder a duas perguntas: i) quais os valores relacionados às produções mensais e anual de serapilheira, e de suas frações constituintes, em fragmento de cerradão localizado no Pontal do Triângulo Mineiro? ii) como variáveis climáticas podem influenciar essas produções?

\section{Material e Métodos}

\section{Área de estudo}

Este estudo foi realizado em um fragmento de cerradão (Figura 1), em área pertencente à Fazenda 
FIGURA 1: Localização da área de estudo no município de Prata, estado de Minas Gerais, Brasil.

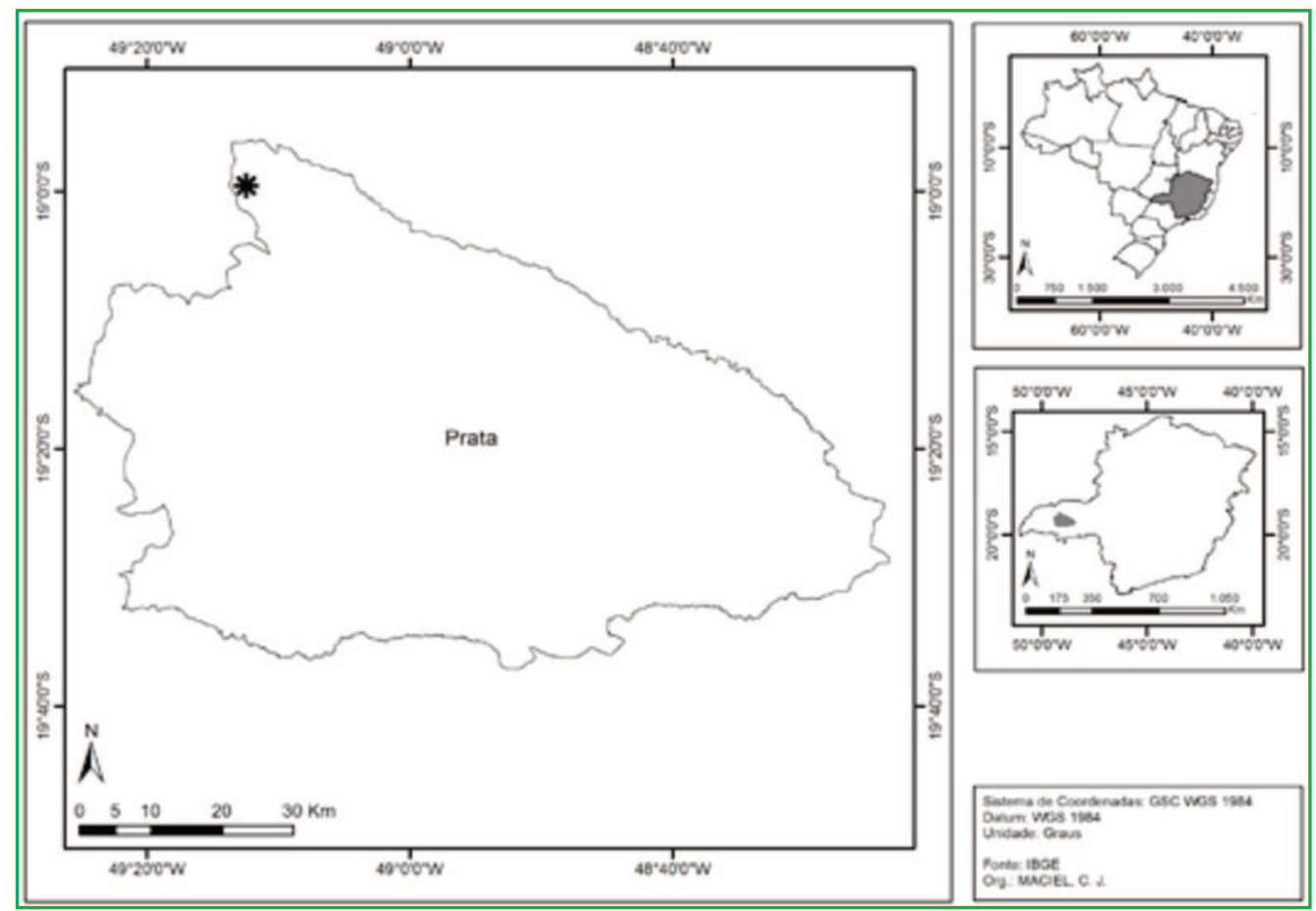

São José, município de Prata (1859'36,67'S e $\left.49^{\circ} 12^{\prime 2} 25,36^{\prime} \mathrm{W}\right)$, no Pontal do Triângulo Mineiro (MG), a $702 \mathrm{~m}$ de altitude. A área possui $9,7 \mathrm{ha}$, e, atualmente, encontra-se rodeada por pastagens.

Ressalta-se que, por força da expansão da fronteira agrícola, remanescentes de vegetação natural localizados na região do Triângulo Mineiro foram reduzidos, e que fragmentos com área superior a 100 ha são muito raros atualmente (CAVALCANTI; JOLY, 2002).

O clima da região foi classificado, segundo o sistema de Köppen, como tipo Aw, caracterizado por verões chuvosos e invernos secos (KOTTEK et al., 2006). No ano estudado (novembro de 2009 a outubro de 2010), a distribuição das chuvas evidenciou a marcante sazonalidade climática característica dessa região, com estação seca de maio a setembro e estação chuvosa de outubro a abril. A precipitação total anual foi de $1.444 \mathrm{~mm}$, sendo que o mês de dezembro foi o mais chuvoso (323 mm) e em agosto não ocorreu precipitação. Setembro foi o mês mais quente, com a média máxima igual a $35,4{ }^{\circ} \mathrm{C}$, e junho o mês mais frio, com média mínima de $12,1^{\circ} \mathrm{C}$ (Figura 2). A velocidade média do vento, ao longo dos meses de estudo, variou entre 0,34 $\mathrm{m} / \mathrm{s}$ (fevereiro) e $1,33 \mathrm{~m} / \mathrm{s}$ (setembro). Nos últimos meses do estudo, a partir de julho, ocorreu maior deslocamento de ar, com variações da velocidade do vento entre $0,85 \mathrm{~m} / \mathrm{s}$ e $1,33 \mathrm{~m} / \mathrm{s}$. Os dados climáticos, provenientes da estação meteorológica de Ituiutaba (OMM: 83521; $560 \mathrm{~m}$ de altitude), localizada a $27 \mathrm{~km}$ da área de estudo, foram obtidos junto ao Instituto Nacional de Meteorologia (INMET). O solo da área de estudo foi definido como latossolo vermelho distrófico (IBGE, 2001).

FIGURA 2: Variação das temperaturas médias (o), mínimas $(\boldsymbol{\Delta})$ e máximas (घ), e da precipitação mensal (barras) em 2009-2010 em Prata (MG), Brasil (fonte: INMET/2012).

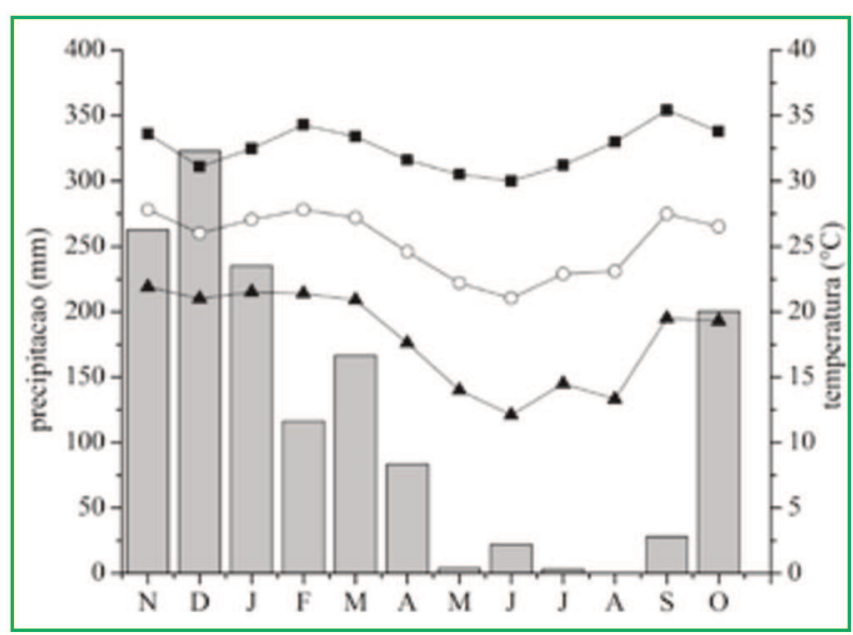




\section{Procedimentos de campo e análises de resultados}

As coletas deste estudo foram realizadas mensalmente entre novembro de 2009 e outubro de 2010. Foram utilizados 20 coletores quadrados, com área de $0,25 \mathrm{~m}^{2}(0,5 \mathrm{~m} \times 0,5 \mathrm{~m})$ e altura de $30 \mathrm{~cm}$ cada um (PROCTOR, 1983), distribuídos em linha, ao longo de uma transecção, mantendo-se uma distância de $8 \mathrm{~m}$ entre os coletores. Foi observada a distância mínima de $50 \mathrm{~m}$ da borda do fragmento, como limite para a instalação dos coletores. Os coletores foram confeccionados com haste de ferro e tela de nylon com malha de $1 \mathrm{~mm}$.

Todo o material coletado foi acondicionado em sacos plásticos numerados para posterior secagem e análise no laboratório de Botânica da Faculdade de Ciências Integradas do Pontal (FACIP), Universidade Federal de Uberlândia (UFU). A triagem manual do material compreendeu a separação da serapilheira total em três frações distintas: a) foliar (folhas); b) fértil (estruturas férteis - botões florais, flores, frutos e sementes, inteiras ou partes destas, desde que reconhecíveis) e c) ramos (gravetos). Nenhuma restrição de diâmetro foi aplicada à fração ramos nas coletas. Após a triagem manual, o material foi acondicionado em sacos de papel, identificados pelo número do coletor e a respectiva fração, e mantido em estufa de circulação forçada de ar, a $70{ }^{\circ} \mathrm{C}$, durante sete dias. Concluída a secagem, foram realizadas pesagens das frações, separadamente, através de balança digital da marca KN WAAGEN, modelo KN 5000.

Os dados obtidos, climáticos e de produção de serapilheira, foram testados quanto à normalidade através do teste de Shapiro-Wilk e, com exceção das temperaturas médias mínimas, todos apresentaram distribuição normal. A transformação logarítmica (ln) foi aplicada aos valores das temperaturas médias mínimas, para a sua utilização nos testes paramétricos. O teste de correlação linear de Pearson $(r)$ foi aplicado para verificar a relação direta entre a produção de serapilheira total, assim como suas frações, e as variáveis climáticas dos meses de estudo (precipitação, temperaturas médias mínimas e máximas e velocidade média do vento). Foram também elaborados gráficos do tipo box-plot, como análise exploratória complementar (ZAR, 1999), utilizando os valores obtidos pelo conjunto de coletores, em cada mês, durante o ano de estudo.

\section{Resultados}

A produção total de serapilheira teve significativa contribuição da fração foliar $(5.068 \mathrm{~kg} / \mathrm{ha})$, representando $67 \%$ da quantidade de serapilheira produzida no ano de estudo. A produção dessa fração foi seguida pela fração ramos $\left(1.652 \mathrm{~kg} \mathrm{ha}^{-1}\right)$ e fração fértil $\left(826 \mathrm{~kg} \mathrm{ha}^{-1}\right)$ (Tabela 1).

A correlação de Pearson indicou a existência da possível influência de componentes climáticos na produção das frações da serapilheira total. A produção mensal de serapilheira, por sua vez, não apresentou valores significativos para a análise de correlação. Os três maiores valores de $r$ obtidos corresponderam à relação entre produção foliar e precipitação e temperaturas médias mínimas, e entre a fração ramos e a velocidade média do vento (Tabela 2). As quantidades da fração ramos, depositadas mensalmente, apresentaram correlação significativa com a velocidade média do vento.

TABELA 1: Valores médios $(\mathrm{kg} / \mathrm{ha})$ para a produção total de serapilheira e das frações foliar, fértil e ramos, com respectivos desvios-padrão, obtidas no período de estudo, no cerradão de Prata (MG), Brasil.

\begin{tabular}{ccccc}
\hline Mês/Ano & Foliar & Fértil & Ramos & Total \\
\hline Nov/09 & $123 \pm 16$ & $149 \pm 39$ & $28 \pm 8$ & $300 \pm 43$ \\
Dez/09 & $247 \pm 27$ & $112 \pm 23$ & $241 \pm 78$ & $599 \pm 79$ \\
Jan/10 & $164 \pm 17$ & $51 \pm 9$ & $96 \pm 34$ & $310 \pm 36$ \\
Fev/10 & $191 \pm 28$ & $85 \pm 31$ & $39 \pm 19$ & $315 \pm 43$ \\
Mar/10 & $349 \pm 31$ & $47 \pm 16$ & $107 \pm 45$ & $504 \pm 64$ \\
Abr/10 & $436 \pm 46$ & $8 \pm 2$ & $43 \pm 21$ & $488 \pm 50$ \\
Mai/10 & $428 \pm 32$ & $6 \pm 4$ & $28 \pm 8$ & $462 \pm 36$ \\
Jun/10 & $618 \pm 56$ & $9 \pm 4$ & $66 \pm 18$ & $692 \pm 64$ \\
Jul/10 & $1.039 \pm 112$ & $38 \pm 6$ & $169 \pm 33$ & $1.246 \pm 138$ \\
Ago/10 & $682 \pm 130$ & $84 \pm 25$ & $224 \pm 89$ & $991 \pm 177$ \\
Set/10 & $510 \pm 105$ & $105 \pm 17$ & $345 \pm 88$ & $960 \pm 143$ \\
Out/10 & $280 \pm 29$ & $132 \pm 26$ & $266 \pm 97$ & $678 \pm 97$ \\
\hline Total & $5.067(67 \%)$ & $826(11 \%)$ & $1.652(22 \%)$ & 7.545 \\
\hline
\end{tabular}


TABELA 2: Valores dos coeficientes de correlação de Pearson $(r)$ entre as produções mensais de serapilheira total (s. total) e suas frações, e as médias das variáveis temperatura máxima e mínima, e velocidade do vento, no período de 11/2009 a 10/2010, em de Prata (MG). Precipitação (Pr); temperaturas médias máximas (TMa); temperaturas médias mínimas (TMi), velocidade média do vento (V). Serapilheira total (Se); folhas (Fo); estruturas férteis (Fe); ramos (Ra).

\begin{tabular}{ccccc}
\hline & Pr & TMa & TMi & V \\
\hline St & $r v=-0,57 ; p>0,05$ & $r=-0,05 ; p>0,05$ & $r=-0,54 ; p>0,05$ & $r=0,57 ; p>0,05$ \\
Fo & $\boldsymbol{r}=\mathbf{- 0 , 7 6 ;} \boldsymbol{p}<\mathbf{0 , 0 1}$ & $r=-0,35 ; p>0,05$ & $\boldsymbol{r}=\mathbf{- 0 , 7 6 ;} \boldsymbol{p}<\mathbf{0 , 0 1}$ & $r=0,27 ; p>0,05$ \\
Fe & $r=0,57 ; p>0,05$ & $\boldsymbol{r}=\mathbf{0 , 6 6} ; \boldsymbol{p}<\mathbf{0 , 0 5}$ & $r=\mathbf{0 , 5 8} ; \boldsymbol{p}<\mathbf{0 , 0 5}$ & $r=0,49 ; p>0,05$ \\
Ra & $r=-0,00 ; p>0,05$ & $r=0,40 ; p>0,05$ & $r=0,06 ; p>0,05$ & $\boldsymbol{r}=\mathbf{0 , 7 0 ;} \boldsymbol{p}<\mathbf{0 , 0 5}$ \\
\hline
\end{tabular}

Os gráficos box-plots (Figura 3) resultaram de valores originados de variações ao longo do ano de estudo, observados para as três frações analisadas da serapilheira total, e, por conseguinte, para a serapilheira total. Contrastando com valores máximos, foram registrados valores iguais a zero como valor mínimo, em diferentes meses de coleta para estruturas férteis e ramos. O valor mínimo zero indica que pelo menos um dos coletores não apresentou as estruturas vegetais consideradas, i.e., estruturas férteis ou ramos.

\section{Discussão}

A produção total de serapilheira foi de 7.546 kg.ha ${ }^{-1}$.ano ${ }^{-1}$ (Tabela 1), valor superior ao encontrado nos cerradões do município de Luiz Antônio, SP (CIANCIARUSO et al., 2006) e de Pirapitinga, MG (GIÁCOMO et al., 2012), que produziram, respectivamente, $5.647 \mathrm{~kg} \cdot \mathrm{ha}^{-1} \cdot$ ano $^{-1}$ e $2.920 \mathrm{~kg}^{-h^{-1}}$. ano

${ }^{1}$. Contudo, o volume de serapilheira da área do presente estudo foi da mesma magnitude do valor de aporte

FIGURA 3: Gráficos box-plots construídos a partir dos valores mensais da produção de serapilheira total, obtidos por 20 coletores, e das frações folhas, estruturas férteis e ramos, no ano de estudo. Médias ( $\square$ ); valores discrepantes (outliers) (ロ); valores mínimos (○).

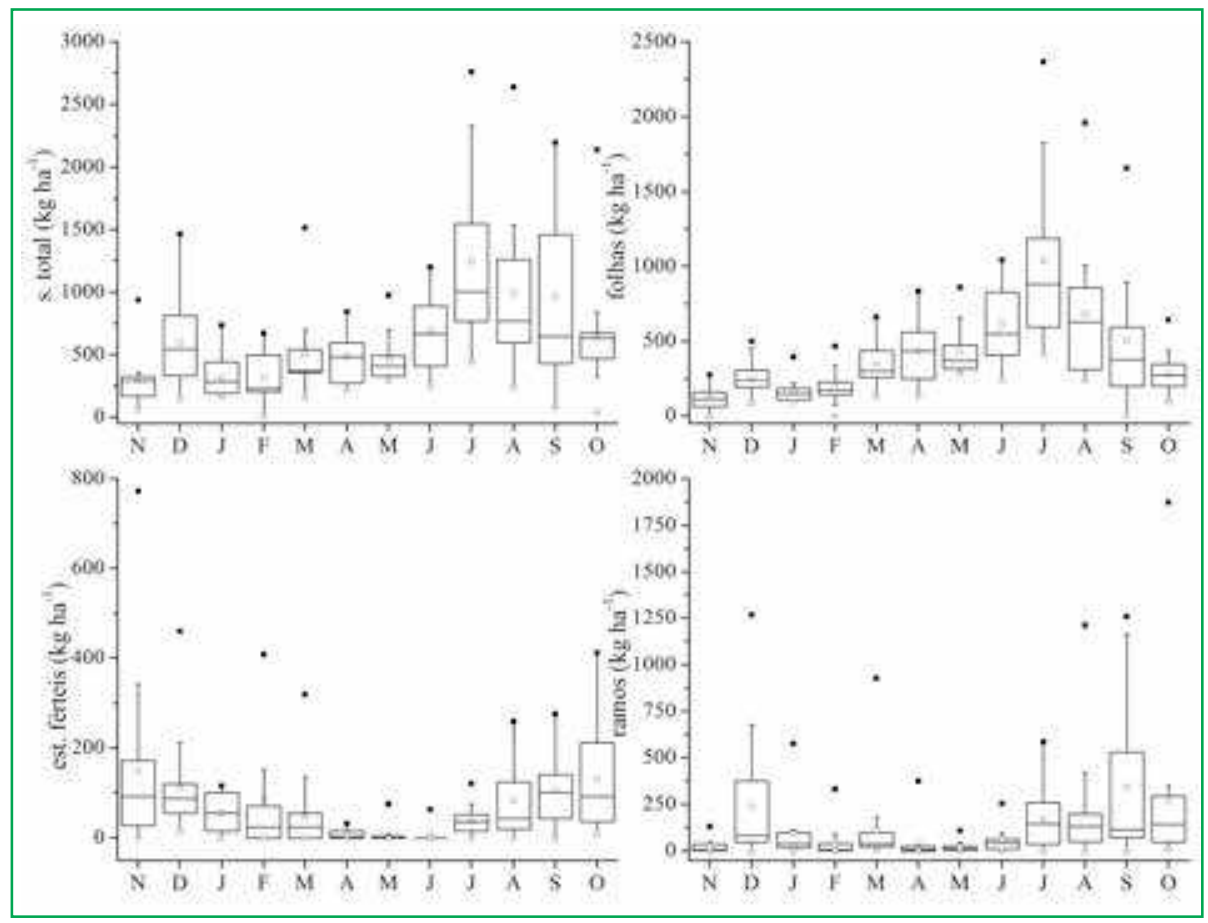


médio anual para os cerradões $(7.800 \mathrm{~kg} / \mathrm{ha})$, informação encontrada em revisão elaborada por Haridasan (2000), referente aos cerrados brasileiros.

A maior quantidade de fração foliar, durante a estação seca (Tabela 1; Figura 2), corroborou estudos que a descreveram como o componente mais importante da serapilheira total em formações florestais e savânicas (CIANCIARUSO et al., 2006; PIRES et al., 2006; SILVA et al., 2007). Esse resultado recebeu importante influência da menor disponibilidade hídrica nos meses de seca, suposição sustentada pela correlação significativa e negativa entre produção da fração foliar e a precipitação $(r=-0,76 ; p<0,01)$. O mesmo valor para o coeficiente de Pearson foi obtido entre produção foliar e temperaturas médias mínimas $(r=-0,76 ; p<0,01)$, indicando provável interferência de baixas temperaturas na produção dessa fração (Tabela 2).

É possível que as citadas correlações tenham resultado de interação sinérgica entre a baixa disponibilidade hídrica e temperaturas mínimas durante o inverno, entre outros fatores. Deve ser considerado ainda que, se cerradões ocupam transições entre os biomas cerrado e floresta estacional tropical (COUTINHO, 2006), sua composição recebe contribuição de elementos florísticos provenientes tanto de formações florestais como de formações savânicas. Desta maneira, elementos florísticos representados por espécies potencialmente sensíveis a estímulos sazonais distintos, como, por exemplo, menor disponibilidade hídrica edáfica e baixas temperaturas, podem estar presentes na comunidade vegetal estudada. Em nosso trabalho foram encontradas as espécies Qualea grandiflora Mart. (Vochysiaceae) e Dipteryx alata Vogel (Fabaceaea), elementos florísticos muito frequentes nos cerrados da região e em boa parte do bioma cerrado (CASTRO et al., 1999; RATTER et al., 2000; 2003). Essas espécies são, provavelmente, mais resistentes ao estresse hídrico e plenamente adaptadas aos profundos e permeáveis solos savânicos (COUTINHO, 2002; FRANCO, 2002). Contudo, é possível que sejam sensíveis à influência de baixas temperaturas (BRANDO; DURIGAN, 2004), embora as temperaturas mínimas observadas em Prata não sejam termoestressantes como as encontradas mais ao sul dessa região, e.g., as registradas no estado de São Paulo, por Silberbauer-Gottsberger et al. (1977). Por sua vez, também foram encontradas no fragmento estudado espécies de ampla distribuição geográfica, como Copaifera langsdorffii Desf. (Fabaceae) e Protium heptaphyllum (Aubl.) Marchand (Burseraceae), além de Platypodium elegans Vogel (Fabaceae). Essas espécies estão presentes em formações florestais estacionais semideciduais e deciduais (RIZZINI, 1997). Presumese que essas três espécies sejam mais sensíveis à menor disponibilidade hídrica edáfica.

A produção de estruturas férteis apresentou relação positiva com temperaturas médias máximas $(r=0,66$; $p<0,05)$ e mínimas $(r=0,58 ; p<0,05)$. Presume-se que a produção dessa fração por espécies encontradas no fragmento também tenha sido influenciada pelos fatores climáticos descritos anteriormente. A menor produção registrada para estruturas férteis foi entre os meses de abril e junho, período anterior ao início da estiagem. Esse resultado teve origem, provavelmente, de comportamento fenológico similar ao observado por Silva et al. (2007) e Valenti et al. (2008), em cerrado localizado em São Carlos (SP) e em cerradão de Nova Xavantina (MT), respectivamente. Os baixos valores para as correlações entre estruturas férteis e componentes climáticos podem ter sido influenciados pela ocorrência de elementos florísticos savânicos, que floresceram e frutificaram em meses de menor umidade (BULHÃO; FIGUEIREDO, 2002), como espécies autocóricas e anemocóricas do cerrado (LENZA; KLINK, 2006). A correlação entre fração ramos e variação do vento foi significativa, apresentando valor elevado para o coeficiente $r(0,70$; $p<0,05)$. Para a produção do componente ramos, além da própria ação do vento, deve ser considerada ainda a influência de chuvas de verão, que contribuem para a queda de galhos e gravetos (DAUBENMIRE, 1974; NEWTON, 2007). Esse fenômeno foi observado por Haase e Hirooka (1998) em remanescentes de cerradão e floresta estacional.

Valores discrepantes nos gráficos box-plots podem indicar variabilidade dos dados obtidos, que pode ter resultado da própria sazonalidade, ou da existência de distintos microambientes no fragmento estudado, assim como pela influência de faixa vegetacional mais 
próxima da borda do fragmento (MURCIA, 1995; GEHLHAUSEN et al., 2000). Não obstante, deve ser considerado que a produção de flores e frutos também pode ter sofrido influência de variações microclimáticas. Perturbações localizadas, pelo eventual deslocamento e herbivoria de bovinos no fragmento, poderiam ter facilitado a ocupação preferencial de microambientes originados por distintas espécies (WILLIAMS-LINERA et al., 1998).

A dissimetria dos dados sustenta a hipótese de variabilidade ambiental, sendo facilmente visualizada pelo deslocamento das medianas (linhas transversais nas caixas da Figura 3) em relação aos quartis, em alguns dos meses estudados. Sobre essa questão, vale ressaltar que nos meses de maio e junho, por exemplo, estruturas férteis foram encontradas apenas em quatro dos 20 coletores utilizados. Também para as outras duas frações, em alguns meses, não foi encontrado material a ser coletado. A assimetria positiva dos dados recebeu considerável contribuição de valores localizados no terceiro quartil. Corrobora essa afirmação a posição da média, nas três frações, superior à mediana na maioria dos meses de estudo, assim como os bigodes (whiskers), ou valores adjacentes, perpendiculares aos quartis (DOWDY et al., 2004; WILCOX, 2009). Os valores adjacentes superiores, isto é, junto ao terceiro quartil, resultaram da distribuição dos valores maiores, limitados a esse quartil (25\%). Os valores adjacentes inferiores foram menos alongados, e seus valores estiveram muitos próximos, ao contrário dos do terceiro quartil. Em alguns meses os whiskers inferiores não ocorreram.

Possíveis perturbações, presentes no fragmento estudado, não teriam sido suficientes para provocar interferências perceptíveis no volume acumulado de serapilheira produzida, especialmente da fração folhas. Não obstante, trabalhos complementares deverão ser desenvolvidos na região para a compreensão da importância e o grau de influência de outros fatores, como características químicas e físicas edáficas, na variação espacial e sazonal da produção de serapilheira em formações savânicas. Assim como, para o entendimento sobre a importância de espécies vegetais do cerrado para a produção mensal e anual de serapilheira, e para o aporte de elementos químicos para o solo após decomposição da serapilheira acumulada.

\section{Agradecimentos}

À Universidade Federal de Uberlândia (UFU), campus Pontal, pelo apoio logístico para o desenvolvimento do trabalho. Aos senhores Sérgio Veraldi e Vilson Oliveira, por terem possibilitado as atividades de campo. Ao discente Conrado José Maciel e ao Prof. Roberto Barboza Castanho, do curso de Geografia da FACIP. Ao Conselho Nacional de Desenvolvimento Científico e Tecnológico (CNPq) e à Fundação de Amparo à Pesquisa do estado de Minas Gerais (FAPEMIG), pelo apoio em diferentes etapas deste trabalho. À Pró-reitoria de Pesquisa e Pósgraduação (PROPP) da UFU, pelos recursos fornecidos referentes ao edital $\mathrm{n}^{-0}$ 04/2009.

\section{Referências}

ADL, S. M. The ecology of soil decomposition. Oxon: CABI Publishing, 2003. 335 p.

BARNES, B. V.; ZAK, D. R.; DENTON, S. R.; SPURR, S. H. Forest ecology. New York: John Wiley and Sons, 1998. 774 p.

BEGON, M.; TOWNSEND, C. R.; HARPER, J. L. Ecology: from individuals to ecosystems. Oxford: Blackwell Publishing. 2006. $738 \mathrm{p}$.

BERG, B.; MCCLAUGHERTY, C. Plant litter: decomposition, humus formation, carbon sequestration. Heidelberg: SpringerVerlag, 2008. 338 p.

BRANDO, P. M.; DURIGAN, G. Changes in cerrado vegetation after disturbance by frost (São Paulo State, Brazil). Plant Ecology, Dordrecht, v. 175, n. 1, p. 205-215, 2004.

BULHÃO, C. F.; FIGUEIREDO, P. S. Fenologia de leguminosas arbóreas em uma área de cerrado marginal no nordeste do Maranhão. Revista Brasileira de Botânica, São Paulo, v. 25, n. 3, p. 361-369, 2002.

CASTRO, A. A. J. F.; MARTINS, F. R.; TAMASHIRO, J. Y.; SHEPHERD, G. J. How rich is the flora of Brazilian cerrados? Annals of the Missouri Botanical Garden, Saint Louis, v. 86, n. 1, p. 192-222, 1999.

CAVALCANTI, R. B.; JOLY, C. A. Biodiversity and conservation priorities in the cerrado region. In: OLIVEIRA, P. S.; MARQUIS, R. J. (Ed.). The cerrados of Brazil: ecology and natural history of a neotropical savanna. New York: Columbia University Press, 2002. p. 351-367.

CHAPIN, F. S. III; MATSON, P. A.; MOONEY, H. A. Principles of terrestrial ecosystem ecology. Nova York: Springer-Verlag, 2002. $436 \mathrm{p}$. 
CHOUDHURY, D. Herbivory induced changes in leaf-litter resource quality: a neglected aspect of herbivory in ecosystem nutrient dynamics. Oikos, Copenhagen, v. 51, n. 3, p. 389-393, 1988.

CIANCIARUSO, M. V.; PIRES, J. S. R.; DELITTI, W. B. C.; SILVA, E. F. L. P. Produção de serapilheira e decomposição do material foliar em um cerradão na Estação Ecológica de Jataí, município de Luiz Antônio, SP, Brasil. Acta Botanica Brasilica, Feira de Santana, v. 20, n. 1, p. 49-59, 2006.

COLEMAN, D. C.; CROSSlEY, D. A. JR.; HENDRIX, P. F. Fundamentals of soil ecology. Burlington: Elsevier, 2004. 386 p.

COUTINHO, L. M. O bioma cerrado. In: KLEIN, A. L. (Ed.). Eugen Warming e o cerrado brasileiro: um século depois. São Paulo: Editora UNESP, 2002. p. 77-91.

COUTINHO, L. M. O conceito de bioma. Acta Botanica Brasilica, Feira de Santana, v. 20, n. 1, p. 13-23, 2006.

DANELL, K.; BERGSTRÖM, R. Mammalian herbivory in terrestrial environments. In: HERRERA, C. M.; PELLMYR, O. (Ed.). Plant-animal interactions: an evolutionary approach. Oxford: Blackwell Science, 2002. p. 107-131.

DAUBENMIRE, R. F. Plants and environment: a textbook of autoecology. New York: John Wiley and Sons, 1974. 422 p.

DENT, D. H.; BAGCHI, R.; ROBINSON, D.; MAJALAP-LEE, N.; BURSLEM, D. F. R. P. Nutrient fluxes via litterfall and leaf litter decomposition vary across a gradient of soil nutrient supply in a lowland tropical rain forest. Plant Soil, Dordrecht, v. 288, n. 1-2, p. 197-215, 2006.

DIDHAM, R. K.; LAWTON, J. H. Edge structure determines the magnitude of changes in microclimate and vegetation structure in tropical forest fragments. Biotropica, Malden, v. 31, n. 1, p. 17-30, 1999.

DOWDY, S.; WEARDON, S.; CHILKO D. Statistics for research. Nova Jersey: John Wiley and Sons, 2004. 627 p.

FRANCO, A. C. Ecophysiology of woody plants. In: OLIVEIRA, P. S.; MARQUIS, R. J. (Ed.). The cerrados of Brazil: ecology and natural history of a neotropical savanna. New York: Columbia University Press, 2002. p. 178-197.

GEHLHAUSEN, S. M.; SCHWARTZ, M. W.; AUGSPURGER, C. K. Vegetation and microclimatic edge effects in two mixedmesophytic forest fragments. Plant Ecology, Dordrecht, v. 147, n. 1, p. 21-35, 2000.

GIÁCOMO, R. G.; PEREIRA, M. G.; MACHADO, D. L. Aporte e decomposição de serapilheira em áreas de cerradão e mata mesofítica na Estação Ecológica de Pirapitinga - MG. Ciência Florestal, Santa Maria, v. 22, n. 4, p. 669-680, 2012.

GUREVITCH, J.; SCHEINER, S. M.; FOX, G. A. Ecologia vegetal. Porto Alegre: Artmed, 2009. 592 p.

HAASE, R.; HIROOKA, R. Y. Structure, composition and small litter dynamics of a semi-deciduous forest in Mato Grosso, Brazil. Flora, Jena, v. 193, p. 141-147, 1998.

HARIDASAN, M. Nutrição mineral de plantas nativas do cerrado. Revista Brasileira de Fisiologia Vegetal, Campinas, v. 12, n. 1, p. 54-64, 2000.

IBGE - INSTITUTO BRASILEIRO DE GEOGRAFIA E ESTATÍSTICA. Mapa de solos do Brasil. Escala 1:5.000.000. Rio de Janeiro: Instituto Brasileiro de Geografia e Estatística, 2001.
KOTTEK, M.; GRISER, J.; BEEK, C.; RUDOLF, B.; RUBEL, F. World map of the Köppen-Geiger climate classification updated. Meteorologische Zeitschrift, Stuttgart, v. 15, n. 3, p. 259-263, 2006.

LATHWELL, D. J.; GROVE, T. L. Soil-plant relationships in the tropics. Annual Review of Ecology, Evolution, and Systematics, Palo Alto, v. 17, p. 1-16, 1986.

LENZA, E.; KLINK, C. A. Comportamento fenológico de espécies lenhosas em um cerrado sentido restrito de Brasília, DF. Revista Brasileira de Botânica, São Paulo, v. 29, n. 4, p. 627-638, 2006.

MURCIA, C. Edge effects in fragmented forests: implications for conservation. Trends in Ecology Evolution, Cambridge, v. 10, n. 2, p. 58-62, 1995.

NEWTON, A. C. Forest ecology and conservation: a handbook of techniques. Oxford: Oxford University Press, 2007. 454 p.

OLIVEIRA, P. S.; MARQUIS, R. J. Introduction: development of research. In: OLIVEIRA, P. S.; MARQUIS, R. J. (Ed.). The cerrados of Brazil: ecology and natural history of a neotropical savanna. New York: Columbia University Press, 2002. p. 1-10.

PIRES, L. A.; BRITEZ, R. M.; MARTEL, G.; PAGANO, S. N. Produção, acúmulo e decomposição da serapilheira em uma restinga da Ilha do Mel, Paranaguá, PR, Brasil. Acta Botanica Brasilica, Feira de Santana, v. 20, n. 1, p. 173-184, 2006.

PROCTOR, J. Tropical forest litterfall. I. Problems of data comparison. In: SUTTON, S. L.; WHITMORE, T. C.; CHADWICK, A. C. (Ed.). Tropical rains forest: ecology and management. Oxford: Blackweel Scientific Publications, 1983. p. 267-273.

RATTER, J. A.; BRIDGEWATER, S.; RIBEIRO, J. F. Analysis of the floristic composition of Brazilian cerrado vegetation III: comparison of the woody vegetation of 376 areas. Edinburgh Journal of Botany, Edinburgh, v. 60, n. 1, p. 57-109, 2003.

RATTER, J. A.; BRIDGEWATER, S.; RIBEIRO, J. F.; DIAS, T. A. B.; SILVA, M. R. Estudo preliminar da distribuição das espécies lenhosas da fitofisionomia cerrado sentido restrito nos estados compreendidos pelo Bioma cerrado. Boletim do Herbário Ezechias Paulo Heringer, Brasília, v. 5, p. 5-43, 2000.

RIUTTA, T.; SLADE, E. M.; BEBBER, D. P.; TAYLOR, M. E.; MALHI, Y.; RIORDAN, P.; MACDONALD, D. W.; MORECROFT, M. D. Experimental evidence for the interacting effects of forest edge, moisture and soil macrofauna on leaf litter decomposition. Soil Biology \& Biochemistry, Oxford, v. 49, p. 124-131, 2012.

RIZZINI, C. T. Tratado de fitogeografia do Brasil: aspectos ecológicos, sociológicos e florísticos. Rio de Janeiro, Âmbito Cultural, 1997. 747 p.

SCHESSL, M.; SILVA, W. L.; GOTTSBERGER, G. Effects of fragmentation on forest structure and litter dynamics in Atlantic rainforest in Pernambuco, Brazil. Flora, Jena, v. 203, p. 215-228, 2008 .

SILBERBAUER-GOTTSBERGER， I.; MORAWETZ, W.; GOTTSBERGER, G. Frost damage of cerrado plants in Botucatu, Brazil, as related to the geographical distribution of species. Biotropica, Malden, v. 9, n. 4, p. 253-261, 1977.

SILVA, C. J.; SANCHES, L.; BLEICH, M. E.; LOBO, F. A.; NOGUEIRA, J. S. Produção de serrapilheira no cerrado e floresta de transição Amazônia-cerrado do centro-oeste brasileiro. Acta Amazonica, Manaus, v. 37, n. 4, p. 543-548, 2007. 
VALENTI, M. W.; CIANCIARUSO, M. V.; BATALHA, M. A. Seasonality of litterfall and leaf decomposition in a cerrado site. Brazilian Journal of Biology, São Carlos, v. 68, n. 3, p. 459-465, 2008.

VAN SCHAIK, C. P.; TERBORGH, J. W.; WRIGHT, S. J. The phenology of tropical forests: adaptive significance and consequences for primary consumers. Annual Review of Ecology, Evolution, and Systematics, Palo Alto, v. 24, p. 353-377, 1993.

WERNECK, M. S.; PEDRALLI, G.; GIESEKE, L. F. Produção de serapilheira em três trechos de uma floresta semidecídua com diferentes graus de perturbação na Estação Ecológica do Tripuí, Ouro Preto, MG. Revista Brasileira de Botânica, São Paulo, v. 24, n. 2, p. 195-198, 2001.
WILCOX, R. R. Basic statistics: understanding conventional methods and modern insights. Oxford: Oxford University Press, 2009. 330 p.

WILLIAMS-LINERA， G.; DOMÍNGUEZ-GASTELÚ， V.; GARCÍA-ZURITA, M. E. Microenvironment and floristics of different edges in a fragmented tropical rainforest. Conservation Biology, San Francisco, v. 12, n. 5, p. 1091-1102, 1998.

ZAR, J. H. Biostatistical analysis. Nova Jersey: Prentice Hall, 1999. $663 \mathrm{p}$. 